\title{
EMD VIA MEMD: MULTIVARIATE NOISE-AIDED COMPUTATION OF STANDARD EMD
}

\author{
NAVEED UR REHMAN \\ Department of Electrical Engineering \\ COMSATS Institute of Information Technology \\ Park Road, Chak Shahzad, Islamabad 44000, Pakistan \\ naveed.rehman@comsats.edu.pk \\ CHEOLSOO PARK \\ Department of Bio Engineering \\ University of California, San Diego, USA \\ charles586@gmail.com \\ NORDEN E. HUANG \\ Research Center for Adaptive Data Analysis \\ National Central University, Zhongli 32001, Taiwan \\ norden@ncu.edu.tw \\ DANILO P. MANDIC* \\ Department of Electrical and Electronic Engineering \\ Imperial College London, Exhibition Road \\ London SW7 2AZ, UK \\ d.mandic@imperial.ac.uk
}

Received 26 April 2013

Accepted 26 April 2013

Published 17 June 2013

\begin{abstract}
A noise-assisted approach in conjunction with multivariate empirical mode decomposition (MEMD) algorithm is proposed for the computation of empirical mode decomposition (EMD), in order to produce localized frequency estimates at the accuracy level of instantaneous frequency. Despite many advantages of EMD, such as its data driven nature, a compact decomposition, and its inherent ability to process nonstationary data, it only caters for signals with a sufficient number of local extrema. In addition, EMD is prone to mode-mixing and is designed for univariate data. We show that the noiseassisted MEMD (NA-MEMD) approach, which utilizes the dyadic filter bank property of MEMD, provides a solution to the above problems when used to calculate standard EMD. The method is also shown to alleviate the effects of noise interference in univariate noise-assisted EMD algorithms which directly add noise to the data. The efficacy of
\end{abstract}

\footnotetext{
${ }^{*}$ Corresponding author.
} 
the proposed method, in terms of improved frequency localization and reduced modemixing, is demonstrated via simulations on electroencephalogram (EEG) data sets, over two paradigms in brain-computer interface (BCI).

Keywords: Empirical mode decomposition; multivariate empirical mode decomposition; electroencephalogram; brain-computer interface.

\section{Introduction}

The Empirical Mode Decomposition (EMD) algorithm expands a given time series $x(k)$ into a set of narrowband oscillatory modes, termed intrinsic mode functions (IMFs), which, unlike the fixed basis functions within the Fourier and wavelet transforms, emerge naturally from the inherent oscillatory modes within the signal $x(k)$ [Huang et al. (1998)]. This offers major advantages in the processing of real world signals, as the basis of EMD (that is, IMFs) are data-adaptive, generic, and much more flexible as compared to the Fourier and wavelet basis functions. Owing to the inherent nonlinearity of the EMD algorithm, this also leads to a compact representation and physically relevant IMFs. Subsequently, the normalized Hilbert transform and the direct quadrature methods can be used to obtain accurate timefrequency (TF) representations [Huang et al. (2009)].

The success of EMD has also highlighted several issues that need further attention, such as the inherent mode-mixing and aliasing. Mode-mixing across the basis functions is mainly manifested by a single IMF either carrying signals of widely disparate scales, or a single mode or scale residing in more than one IMF [Wu and Huang (2009)]. This is a consequence of signal intermittency, causing the overlapping of IMF spectra and aliasing in the TF domain. The mode-mixing phenomenon compromises the physical meaning of IMFs by, for instance, erroneously suggesting different physical processes present in a single IMF.

The two sources of aliasing are: (1) the extrema sampling, a key step in EMD filtering, which has been shown to be a sub-Nyquist process; (2) the equivalent interpolation filter of EMD (e.g. a cubic spline) which is far from an ideal low-pass filter, resulting in further aliasing. It is important to highlight that the EMD filtering process critically depends on the sampling of signal extrema and, therefore, the presence of a sufficient number of extrema in an input is a prerequisite for the initiation and the successful operation of EMD. For instance, standard EMD fails to operate on a Dirac pulse, since there are not enough extrema for a meaningful IMF. To obtain an equivalent impulse response of an EMD filter for its characterization, Flandrin et al. considered an idealized Dirac pulse as the limit of a noisy pulse and performed an ensemble average of $N$ such realizations [Flandrin et al. (2005)]. The addition of noise to the Dirac pulse introduces additional extrema to the signal, which helps to initiate the EMD filtering process and to obtain its equivalent impulse response.

The idea of adding noise ensemble to the signal also underpins the ensemble empirical mode decomposition (EEMD) algorithm [Wu and Huang (2009)], 
which operates by adding multiple independent noise realizations to data, applies EMD to every member of the resulting ensemble, and finally performs ensemble average of each set of IMFs to obtain an enhanced decomposition. In doing so, EEMD makes use of the dyadic filter bank property of EMD when applied to white Gaussian noise (WGN); subsequent averaging over the noise ensemble benefits from the so induced large number of extrema, and yields more localized inherent modes present in the data, in addition to the decomposition which is almost free from mode-mixing. However, a consequence of adding noise directly to the data is that a trace of residual noise is likely to remain in the IMFs. The amplitude (power) of this residuum depends on the number of realizations averaged (size of ensemble), thus, compromising the "completeness" of the retained signal. Several modifications of EEMD have been proposed to deal with this problem; the approach in Torres et al. [2011] adds a carefully chosen noise at each decomposition stage to compute a unique residual; the complementary ensemble empirical mode decomposition (CEEMD) removes the residue due to WGN, via pairs of complementary ensemble IMFs, obtained from the positive and negative portions of the added noise [Yeh et al. (2010)]. Both algorithms, however, still add noise directly to the input data and, hence, cannot guarantee the "completeness" of the reconstructed signal in the strict sense; they are also computationally more expensive than the EEMD method.

To address the above issues, the recently proposed noise-assisted multivariate empirical mode decomposition (NA-MEMD) [Rehman and Mandic (2011)] employs multivariate extensions of EMD (MEMD) [Rehman and Mandic (2010b); Rilling et al. (2007); Altaf et al. (2007)], that have been originally designed for signals containing multiple data channels and have shown significant potential in data fusion [Looney and Mandic (2009)], phase synchronization [Looney and Mandic (2009)], and frequency localization [Rehman and Mandic (2010a)]. The NA-MEMD operates by first creating a multivariate signal consisting of one or more input data channels and adjacent independent realizations of WGN in separate channels. The resulting multivariate signal, comprising data and noise channels, is processed using the MEMD method, and the IMFs corresponding to the original data are reconstructed to yield the desired decomposition [Rehman and Mandic (2011)]. In this way, unlike EEMD, the physically disjointed input and noise subspaces within NA-MEMD prevent direct noise artifacts. Notwithstanding the differences between the EEMD and NA-MEMD, the main rationale behind both methods remains the same: making use of the dyadic filter bank structure of (M)EMD for WGN for improved performance of the standard univariate EMD.

In this work, we set out to assess the potential of NA-MEMD in the processing of both univariate and multivariate EEG signals, which are the most convenient means to measure neurophysiological activity due to their noninvasive nature and inexpensive recording equipment. Brain-computer interface (BCI), an emerging technology focusing computer-aided control using brain activity, is based on EEG data and has found diverse applications ranging from bioengineering to neuroprosthetics. From 
the signal processing perspective, we provide a rigorous analysis of the usefulness of NA-MEMD for the computation of EMD, leading to the justification for its use for both univariate and multivariate time series. The analysis relates the power of the channels within the input noise subspace and the degree of localization provided by the algorithm, highlighting the robustness and flexibility of the proposed approach. The mode-alignment and filter bank properties of NA-MEMD are also analyzed, together with its equivalent impulse response. The advantages of the NA-MEMD method over standard EMD and EEMD, for the analysis of univariate data, are illustrated for these key problems of standard EMD: frequency localization, noise interference, and mode-mixing. Furthermore, we employ NA-MEMD in EEG analysis and illustrate its ability to account for the nonstationarity, multichannel nature, inherent drift in frequency estimation, and to overcome the uncertainty in the estimation associated with methods employing fixed basis function. These virtues of NA-MEMD are shown over two case studies: steady state visual evoked potential (SSVEP) and motor imagery EEG data paradigms.

The paper is organized as follows: Secs. 2 and 3 describe the background of EMD and MEMD related algorithms respectively. Section 4 presents an extensive set of simulations on real and synthetic data, comparing the NA-MEMD with EMD and EEMD. Finally, applications of NA-MEMD on nonstationary EEG signals are presented, followed by the discussion and the conclusions.

\section{Empirical Mode Decomposition and Its Noise-Aided Extensions}

EMD is a data-driven technique to decompose a signal, by means of an iterative process called the sifting algorithm, into a finite set of oscillatory components called IMFs [Huang et al. (1998)]. These IMFs represent the temporal modes (scales) present in the data. For a time series $x(k)$, the EMD represents a sum of IMFs $\left\{c_{m}(k)\right\}, m=1, \ldots, M$ and the residual $r(k)$, that is

$$
x(k)=\sum_{m=1}^{M} c_{m}(k)+r(k),
$$

where the residual $r(k)$, unlike $\left\{c_{m}(k)\right\}_{m=1}^{M}$, does not contain any oscillations and its physical meaning is a trend within the signal.

However, the EMD output is prone to mode-mixing which mainly occurs due to the overlapping of the IMF spectra [Huang et al. (1998)], and the aliasing caused by sub-Nyquist extrema sampling. To overcome these issues, the ensemble EMD (EEMD) algorithm [Wu and Huang (2009)], makes use of the dyadic filter bank property of EMD for WGN by adding noise ensembles to the input data, before applying EMD. This way, EEMD effectively populates the whole TF space. The IMFs are then obtained from the ensemble mean of the corresponding IMFs (obtained from noise ensemble added to input). More specifically, if $x(t)$ denotes the input signal, and $w_{n}(t)$ the $n$th realization of WGN, then the ensemble of the 


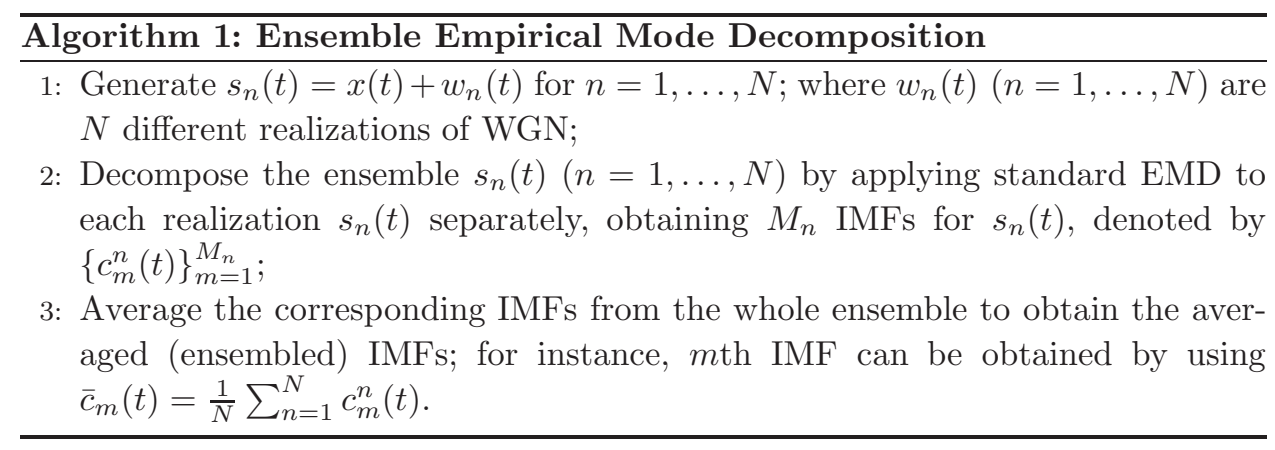

input signal and WGN can be expressed as

$$
\left\{s_{n}(t)\right\}_{n=1}^{N}=x(t)+\left\{w_{n}(t)\right\}_{n=1}^{N}
$$

for $1 \leq n \leq N$, where $N$ is the total number of the ensemble members used in the process. The EEMD algorithm is described in Algorithm 1.

Statistically, the effect of added WGN within the set $s_{n}(t)$ cancels out when taking the mean over a sufficiently large ensemble, in accordance with the following well-established rule:

$$
\gamma_{n}=\frac{\eta}{\sqrt{N}}
$$

where $\eta$ is the amplitude of the added noise, and $\gamma_{n}$ is the standard deviation of the error signal between the final output from EEMD and the original signal $x(t)$.

The relation (3) highlights a significant computational drawback in EEMD: to completely cancel the effect of added noise from the output, that is to ensure completeness of EEMD, an infinitely large number of ensemble members must be considered.

\section{Multivariate EMD and Its Noise-Aided Extensions}

\subsection{Multivariate empirical mode decomposition}

Multivariate empirical mode decomposition (MEMD) algorithm was designed to operate for an arbitrary number of input channels [Rehman and Mandic (2010b); Rilling et al. (2007); Altaf et al. (2007)]. Similarly to EMD, the output of the MEMD for WGN exhibits a quasi-dyadic filter bank structure [Rehman and Mandic (2011); Flandrin and Goncalves (2004); Flandrin et al. (2005)]. This, together with minimal cross-channel leakage in MEMD, ensures enhanced identification of intrinsic oscillatory modes within a signal. Generalizing (1), given a $p$-variate signal $\mathbf{s}(t)$, MEMD produces $M$ multivariate IMFs:

$$
\mathbf{s}(t)=\sum_{m=1}^{M} \mathbf{c}_{m}(t)+\mathbf{r}(t)
$$


where $\mathbf{c}_{m}(t)$ represents the $m$ th IMF of $\mathbf{s}(t)$ (also $p$-variate) and $\mathbf{r}(t)$ denotes the $p$-variate residual.

Within MEMD, estimation of the local multivariate mean is a key step which is performed by taking multiple real-valued projections of the original signal. The extrema of such single-dimensional projections are then interpolated componentwise to yield multi-dimensional signal envelopes. Multiple signal projections are necessary to model higher dimensional fields, this makes it possible to operate in the domain, such as the complex $\mathbb{C}$ and quaternion $\mathbb{H}$ fields, which are not ordered $^{\mathrm{a}}$ [Mandic and Goh (2009)]. The so projected envelopes are averaged to give an estimate of the local mean. More specifically, if $e_{\left\{\theta_{1}, \theta_{2}, \ldots, \theta_{n-1}\right\}}$ denotes the envelope in the direction represented by a vector $\theta=\left\{\theta_{1}, \theta_{2}, \ldots, \theta_{n-1}\right\}$ in $\mathbb{R}^{n}$, then the local mean $m(t)$ of a signal $\mathbf{s}(\mathbf{t})$ can be estimated by using

$$
\begin{aligned}
m(t) & =\frac{1}{2 \pi^{n-1}} \int_{\theta_{1}=0}^{\pi} \int_{\theta_{2}=0}^{\pi} \cdots \int_{\theta_{n-1}=0}^{2 \pi} e_{\left\{\theta_{1}, \theta_{2}, \ldots, \theta_{n-1}\right\}} d \theta_{1} d \theta_{2} \cdots d \theta_{n-1}, \\
& \approx \frac{1}{V_{1} V_{2} \cdots V_{n-1}} \sum_{v_{1}=1}^{V_{1}} \sum_{v_{2}=1}^{V_{2}} \cdots \sum_{v_{n-1}=1}^{V_{n-1}} e_{\left\{\theta_{v_{1}}, \theta_{v_{2}}, \ldots, \theta_{v_{n-1}}\right\}},
\end{aligned}
$$

where the set $\left\{V_{1}, V_{2}, \ldots, V_{n-1}\right\}$ denotes the number of direction vectors taken along the directions $\left\{\theta_{1}, \theta_{2}, \ldots, \theta_{n-1}\right\}$ respectively.

The accuracy of the above approximation is dependent on the choice of the set of direction vectors, as was shown in Cui and Freeden [1997]. To represent direction vectors in an $n$-dimensional $(n \mathrm{D})$ space, for convenience of presentation, sample points on the surface of corresponding unit $(n-1)$-spheres are considered. ${ }^{\mathrm{b}}$ In MEMD, those points are generated by the low-discrepancy Hammersley sequences in $\mathbb{R}^{n}$, ensuring more efficient and accurate implementation of the integral in (6), for a finite point set [Niederreiter (1992)]; details on how to compute the Hammersley sequence and the listing of the MEMD algorithm is given in Appendix A. Despite its merits when processing multivariate nonstationary signals, MEMD inherits a degree of mode-mixing, which motivated the development of NA-MEMD.

\subsection{Noise-assisted multivariate EMD}

The NA-MEMD algorithm attempts to eliminate the interference of noise in EEMD and reduce the mode-mixing in both EMD and MEMD outputs; it is outlined in Algorithm 2, whereby the first step ensures that the noise channels are not added to signals which already fulfill the IMF criteria. The method operates by forming a multivariate signal consisting of input data and noise in separate channel(s) [Rehman and Mandic (2011)]. The MEMD is then applied to the so constructed

\footnotetext{
aFor instance, operations such as, "greater than" $>$ and "less than" $<$ do not exist in $\mathbb{C}$ and $\mathbb{H}$.

${ }^{\mathrm{b}}$ An $n$-sphere, or equivalently an $(n-1)$-dimensional hypersphere, can be considered as an extension of the ordinary sphere to an arbitrary dimension.
} 


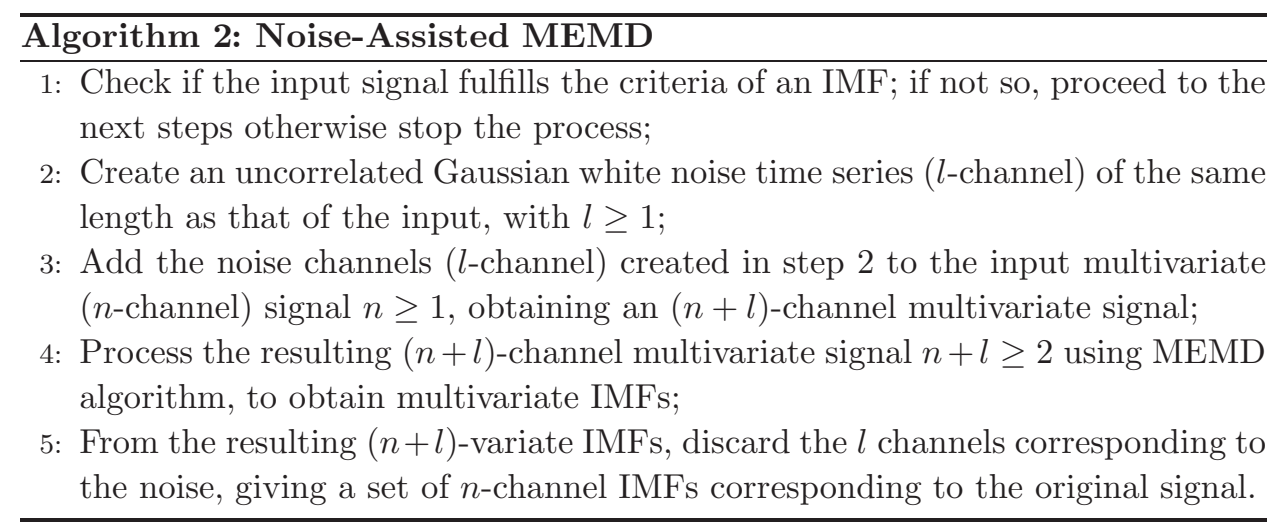

multivariate signal and the resulting IMF subspace corresponding to WGN is discarded. The desired separation between the input and noise diminishes noise interference in the output, owing to the minimal cross-channel leakage in NA-MEMD. Since the added noise channels occupy a broad range in the frequency spectrum, MEMD aligns its different components (IMFs) in accordance with the quasi-dyadic filter bank structure, with each component carrying a separate frequency sub-band of the original signal. In doing so, the IMFs corresponding to the original data also align themselves in accordance with the quasi-dyadic filter bank structure. ${ }^{\mathrm{c}}$ This, in turn, helps to reduce the mode-mixing problem within the extracted IMFs.

The sifting process employed in MEMD and NA-MEMD can be stopped when all the projected signals fulfill an EMD stopping criterion. In the simulations presented in this paper, we use a version of the stopping criterion, proposed in Rilling et al. [2003], which ensures that the outputs of MEMD and NA-MEMD follow a quasidyadic filter bank structure and defines an evaluation function based on the envelope amplitude of an input as:

$$
\mathbf{a}(t)=\frac{1}{V} \sum_{v=1}^{V}\left|\mathbf{e}_{\theta_{v}}(t)-\mathbf{m}(t)\right| .
$$

The sifting process is continued until the the evaluation function, $f(t)=\left|\frac{\mathbf{m}(t)}{\mathbf{a}(t)}\right|$ falls below some predefined thresholds $\left[\begin{array}{lll}\sigma_{1} & \sigma_{2} & \alpha\end{array}\right]$, where $\mathbf{m}(t)$ is the local mean signal.

\subsubsection{Effect of noise power}

The behavior of the NA-MEMD algorithm changes according to the power level of the added noise channels, with the algorithm performing similarly to standard EMD for infinitesimally small noise amplitudes. Increasing the noise power would further enforce the quasi-dyadic filter bank structure on input data. However, excessive

${ }^{\mathrm{c}}$ This is illustrated by an example in the next section. 
noise levels can compromise the data-driven ability of the (M)EMD-based algorithms. A rule of thumb is to choose the variance (power) of noise within $2-10 \%$ of the variance of the input. The noise with power above this range may result in an unnecessary mode-mixing in the output (see Fig. 7 and the corresponding discussion).

\subsubsection{Ensemble NA-MEMD and its equivalent impulse response}

Since the NA-MEMD method employs a single realization of $l$-channel Gaussian noise for its operation, it may yield slightly different outputs for different trials of $l$-channel WGN, even if same statistics are employed in all trials. To overcome this uniqueness problem, an ensemble approach similar to EEMD can be adopted, whereby instead of using a single realization of $l$-channel noise, an ensemble of independent $l$-channel noise realizations can be considered, in a manner similar to EEMD. The set of multivariate IMFs obtained from MEMD are then ensemble averaged to obtain the output. Although the ensemble NA-MEMD method uses the approach similar to that of EEMD, it does not suffer from the inherent EEMD problem - the noise interference in the output data - since noise is never added to input directly, but occupies a disjointed noise space. Similarly to EEMD, the output of ensemble NA-MEMD may not strictly conform to the definition of an IMF since an ensemble average of multiple IMFs may not satisfy the conditions of an IMF. However, the benefits gained by taking multiple noise realizations, in terms of accurate TF representation and reduced mode mixing, normally outweigh this drawback.

The equivalent impulse response of NA-MEMD is next established by using a discrete version of the Dirac impulse:

$$
\delta[n]= \begin{cases}1 & \text { for } n=0 \\ 0 & \text { for } n \neq 1\end{cases}
$$

Note that since the operation of EMD requires the existence of a sufficient number of extrema in a signal, finding its impulse response is hard given only one maximum in $\delta[n]$. Through NA-MEMD, the required extrema are introduced via the noise subspace, to initiate the EMD algorithm.

In Fig. 1, the impulse response of the NA-MEMD algorithm is shown for the input data length of $K=256, l=2$ dimensional noise subspace and $N=100$ independent realizations of WGN. Specifically, the original Dirac impulse function is shown (first row) along with the first five ${ }^{\mathrm{d}}$ ensemble-averaged IMFs. Notice the similarity of these IMFs with those obtained by the wavelet analysis or EEMD approach [Flandrin and Goncalves (2004)]. Observe that the averaged IMFs have the same shape for each index $m$ suggesting a self-similar structure observed previously

\footnotetext{
${ }^{d}$ The noise channels and their respective decompositions are not shown here for easy visualization.
} 


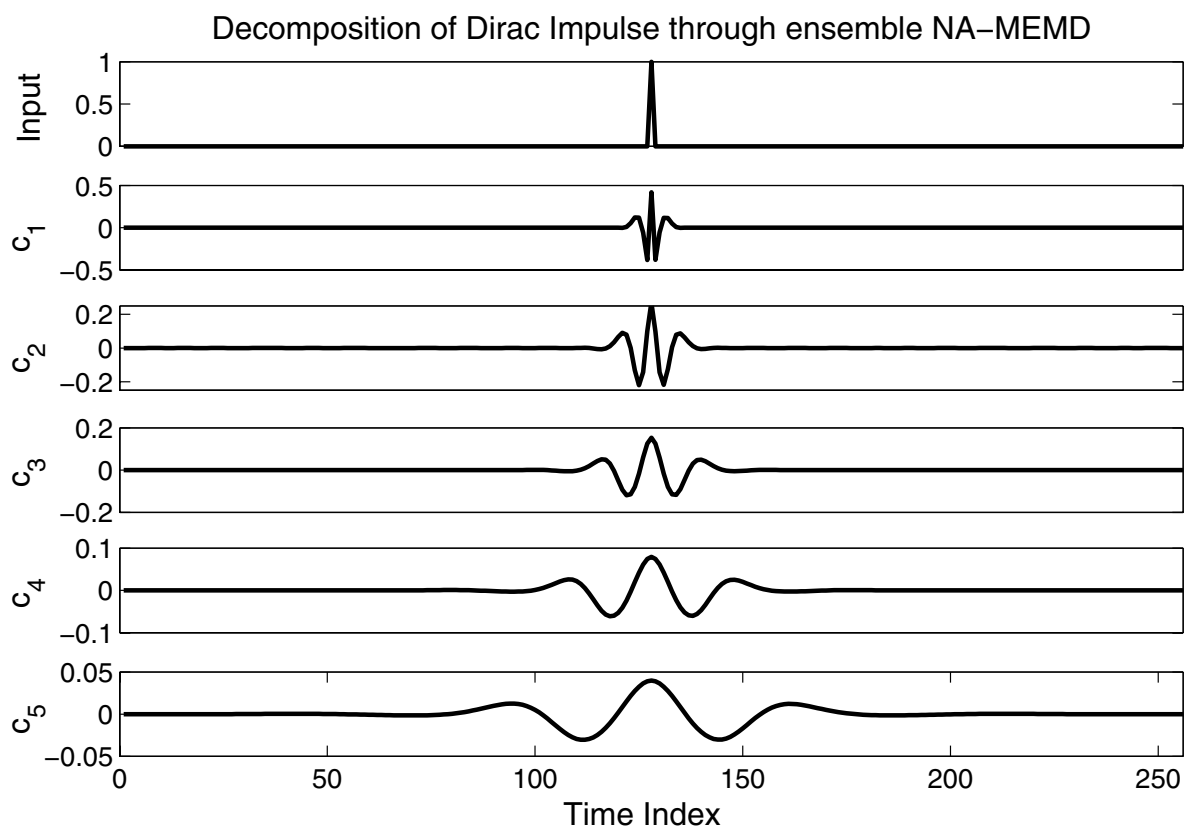

Fig. 1. Decomposition of a discrete-time Dirac impulse via the NA-MEMD algorithm.

for EMD and MEMD-based decompositions [Flandrin et al. (2005); Rehman and Mandic (2011)].

\subsubsection{Mode-alignment and quasi-dyadic filter bank property}

The filter bank property of MEMD in the case of WGN is well established [Rehman and Mandic (2011)]. Moreover, MEMD has also been shown to align common oscillatory modes from an input data; Fig. 2 shows the magnitude of cross correlations calculated between normalised IMFs of two channels of the input bivariate WGN of length $L=1000$, averaged over $N=1000$ realisations, with bivariate EMD (left) outperforming standard EMD applied channel-wise (right). The normalisation of IMFs was performed independently on each channel. The cross-correlation estimates $\Upsilon\left(m, m^{\prime}\right)$ were calculated for IMFs obtained from MEMD and standard EMD:

$$
\Upsilon\left(m, m^{\prime}\right)=\left|\frac{1}{N} \sum_{j=1}^{N} \frac{\Upsilon^{j}\left(m, m^{\prime}\right)}{\sqrt{\Upsilon^{j}(m, m) \Upsilon^{j}\left(m^{\prime}, m^{\prime}\right)}}\right|,
$$

where

$$
\Upsilon^{j}\left(m, m^{\prime}\right)=\frac{1}{K} \sum_{k=1}^{K} c_{m}^{j}(k) c_{m^{\prime}}^{j}(k)
$$

and $c_{m}^{j}(k)$ is the $m$ th IMF corresponding to the $j$ th noise ensemble. 
MEMD mode alignment for WGN

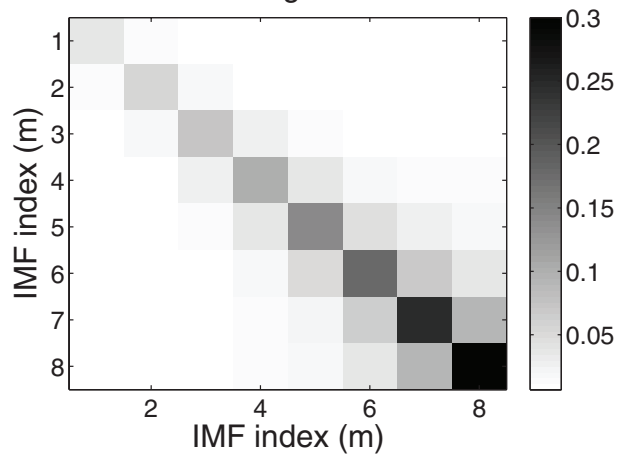

(a)

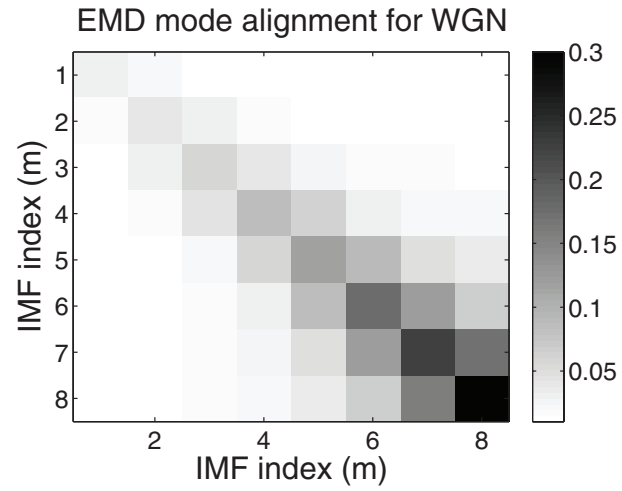

(b)

Fig. 2. The magnitudes of cross correlations calculated between normalised IMFs of two channels of the input bivariate WGN obtained from: (a) BEMD and (b) standard univariate EMD channelwise.

Figure 2(a) shows that owing to the filter bank structure of MEMD, a significant overlapping of the spectra of corresponding (same-indexed) IMFs from multiple channels is observed, resulting in cross-correlation estimates having comparatively larger values along the diagonal $\left(m=m^{\prime}\right)$. In the EMD-based decomposition, however, significant values of the cross-correlation estimates are observed off-diagonal $\left(m \neq m^{\prime}\right)$, indicating miss-aligned IMFs (and spectral leakage), as visible in Fig. 2(b).

Benefiting from the mode-alignment within MEMD, NA-MEMD can be used to reduce the mode-mixing in standard EMD. Indeed, by enforcing the quasi-dyadic filter bank structure on input data through the noise channels, spectra from different IMFs are expected to be more localized in the frequency domain, thus, reducing the mode-mixing problem.

Figures 3 and 4 show the ability of noise-aided (M)EMD algorithms to reduce mode-mixing in univariate EMD. In Fig. 3, the EMD based decomposition of a synthetic signal comprising of three different tones is shown: two low-frequency tones $(0.23 \mathrm{~Hz}$ and $1.0 \mathrm{~Hz})$ were summed together along with a high-frequency sinusoid $(2 \mathrm{~Hz})$ added between samples 1,000 and 1,650. In the noise sub-space within MEMD, two noise channels, $l=2$, were used corresponding to the SNR of $17 \mathrm{~dB}$, and $N=200$ independent noise ensembles were employed. The number of directions used in $\mathrm{MEMD}^{\mathrm{e}}$ were $V=64$ and the parameters of the stopping criterion used in both EMD and MEMD were $\left[\sigma_{1}=0.05, \sigma_{2}=0.5, \alpha=0.05\right]$. For EEMD, the number of noise realizations were taken as 1,000 with signal-to-noise channel power ratio of $7 \mathrm{~dB}$.

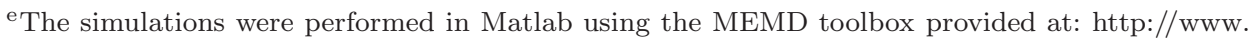
commsp.ee.ic.ac.uk/ mandic/research/emd.htm. 
(a)

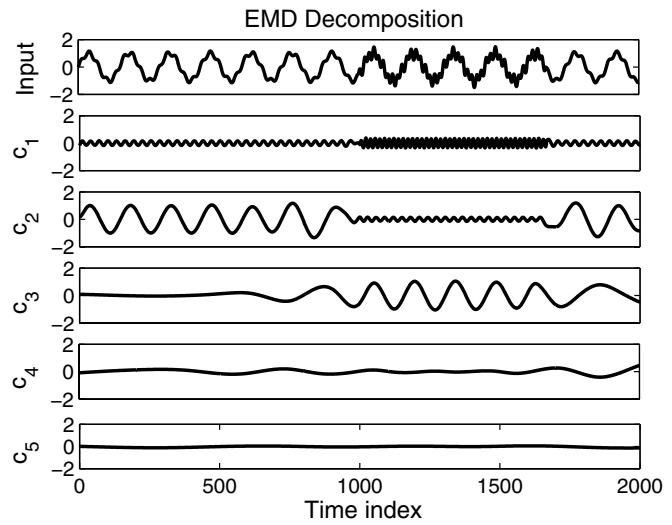

(b)

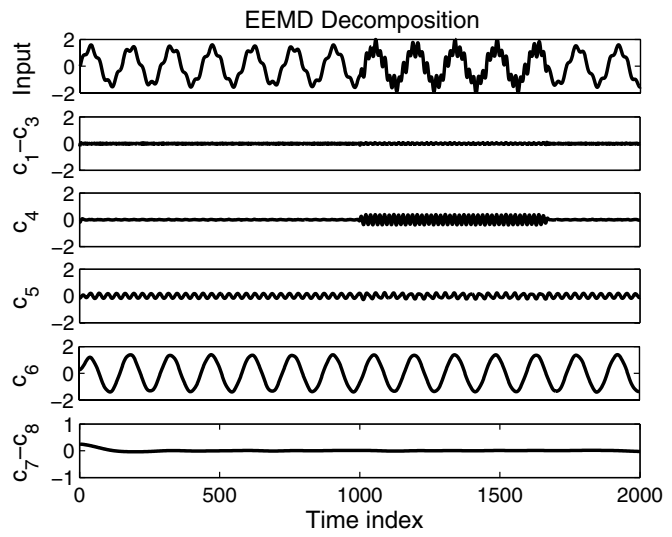

(c)
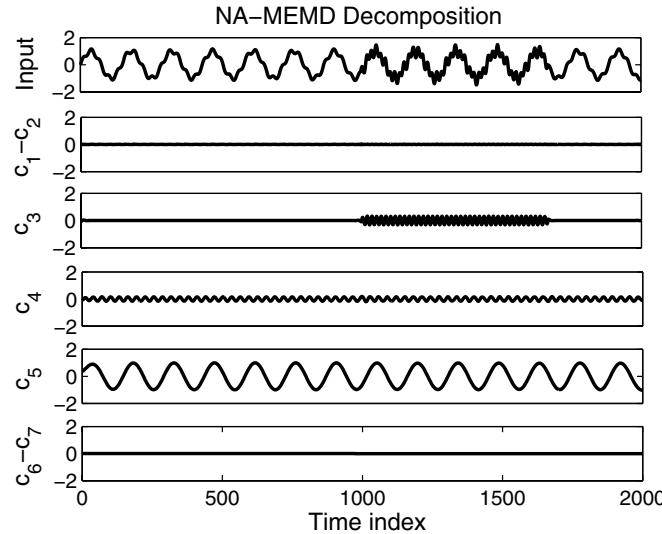

Fig. 3. Advantages of NA-MEMD in reducing mode-mixing. IMFs of a synthetic signal obtained by applying (a) standard EMD, (b) EEMD, and (c) NA-MEMD. 
(a)

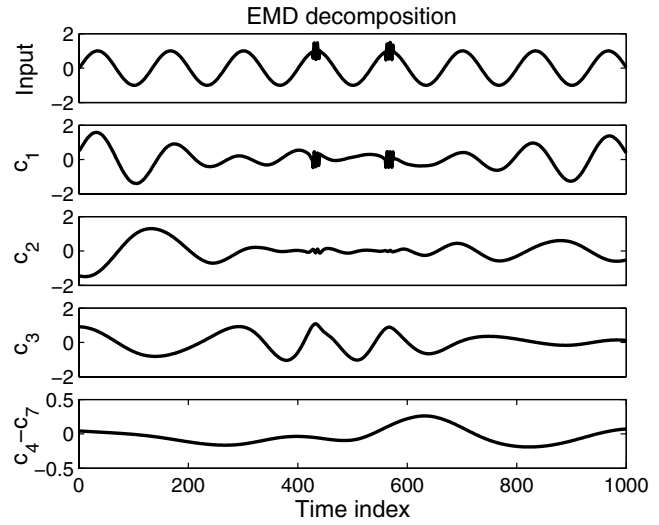

(b)

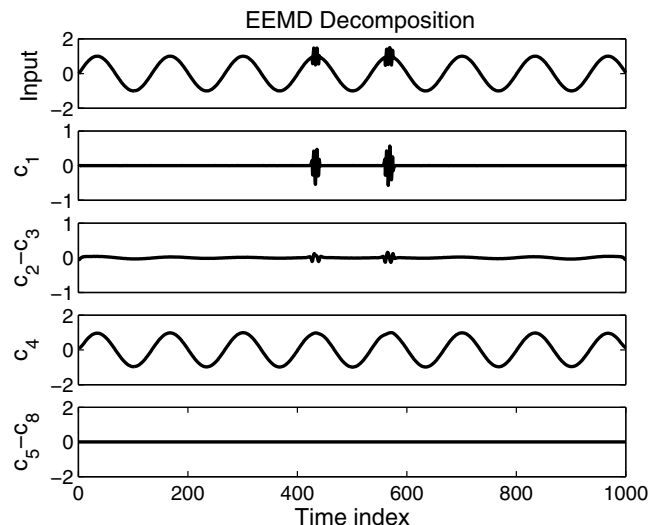

(c)

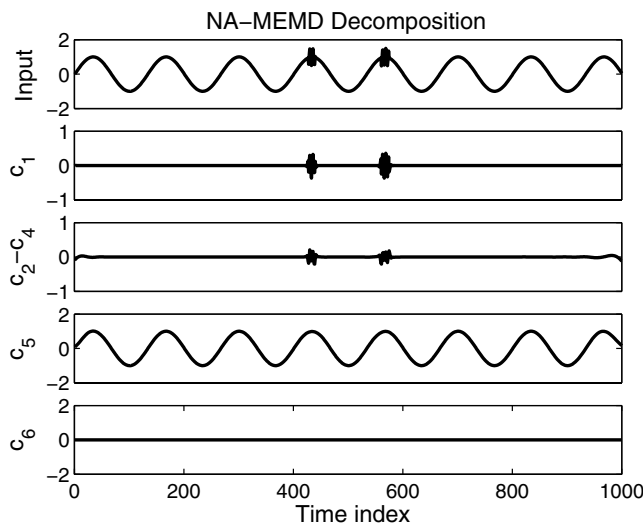

Fig. 4. Advantages of NA-MEMD in reducing mode-mixing. IMFs of a synthetic signal obtained by applying (a) standard EMD, (b) EEMD, and (c) NA-MEMD. 
The resulting signal and its standard EMD based decomposition is shown in Fig. 3(a). Mode-mixing is evident in $c_{1}$ and $c_{2}$ which contains multiple modes (scales). The IMFs from EEMD and NA-MEMD applied to the resulting trivariate signal (original data and two noise channels) are shown in Figs. 3(b) and 3(c) respectively. Observe that the IMFs are now free from mode-mixing, as all the tones are decomposed as separate $\operatorname{IMFs}\left(c_{4}, c_{5}\right.$, and $c_{6}$ in EEMD and $c_{3}, c_{4}$, and $c_{5}$ in NA-MEMD).

Similarly, Fig. 4 shows the decompositions of a different synthetic signal obtained from EMD (a), EEMD (b), and NA-MEMD (c). The input signal consists of a $2 \mathrm{~Hz}$ sinewave corrupted by an intermittent interference between samples 400-600. Ideally, a perfectly localized sinewave should occupy a single IMF, as it satisfies the IMF conditions, however, for the case of EMD, mode mixing occurred in $c_{1}$ and $c_{3}$. On the other hand, the noise-assisted EEMD and NA-MEMD were able to perfectly localize the single-scale sinewave. For NA-MEMD, $l=2$ additional noise channels were used to create a trivariate signal, with the signal-to-noise channel power ratio of $17 \mathrm{~dB}$. For EEMD, the number of noise realizations were taken as 1,000 with signal-to-noise channel power ratio of $7 \mathrm{~dB}$.

\section{NA-MEMD Versus EEMD}

Unlike EEMD, in NA-MEMD noise is not added directly to the input signal. Instead it is kept in separate channels of a multivariate signal and processed directly using MEMD. This way, the output decomposition has no additive noise artifacts, same number of IMFs are guaranteed for each data channel, and similar oscillatory modes are located in same-indexed IMFs, a property not possible to achieve using the standard EMD and EEMD. Moreover, since NA-MEMD is based on multivariate EMD, it can handle both univariate and multivariate signals, whereas EEMD, by design, can only cater for univariate signals.

In the following, a comparison of the completeness of the two methods is investigated, with the amount of residual noise in the reconstructed signal and their sensitivity to input noise power used as the performance metric.

\subsection{Residual noise and sensitivity to noise power}

Residual noise in EEMD is prominent because it is added directly to the signal in order to enforce the dyadic filter bank structure. This compromises the completeness of the method for a finite noise ensemble, albeit its effects can be reduced by increasing the number of noise ensembles $N$, in accordance with (3). On the other hand, in NA-MEMD, noise does not directly interfere with the original signal as the noise subspace occupies separate channels to those containing original data, that is, the signal and noise subspaces do not overlap.

Figure 5 verifies these properties for the synthetic signals shown in Figs. 3 and 4. In Fig. 5, the average power of the error signal is plotted as a function of a ratio between the noise and the signal power, evaluated for the signals from Fig. 3(dashed) 


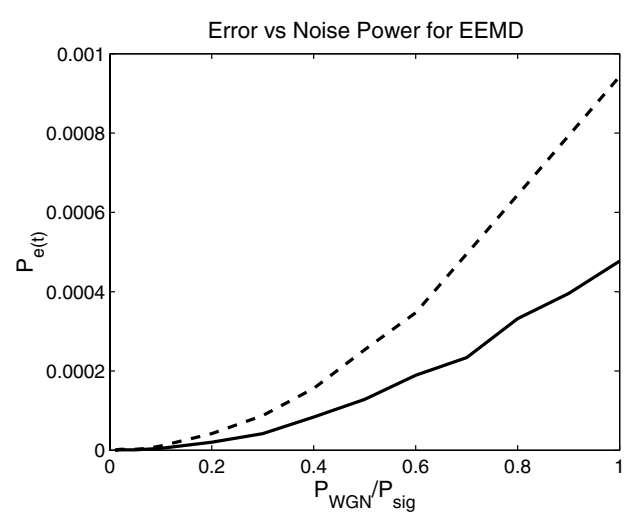

(a)

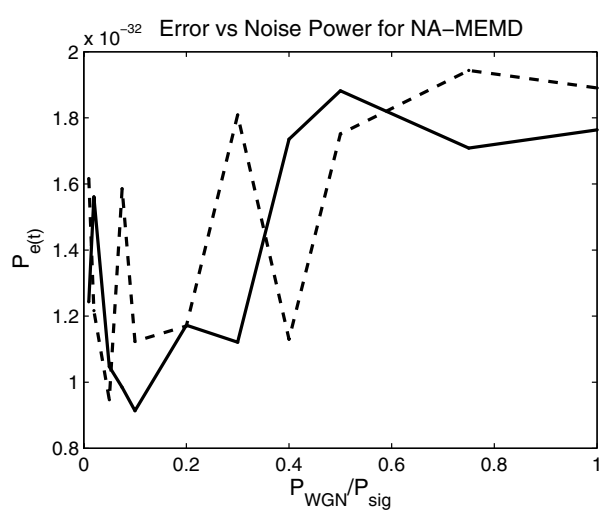

(b)

Fig. 5. Sensitivity to added noise power of EEMD and NA-MEMD. Plots represent the average power of the error signal as a function of the noise to signal power in the case of (a) EEMD and (b) NA-MEMD, for the input signals shown in Fig. 3 (dashed line) and Fig. 4 (thick line).

and Fig. 4(thick), using both EEMD (a) and NA-MEMD (b). In the case of EEMD, as expected, the power of the error signal, which is an indicator of residual noise level, increased with the added noise power. On the other hand, residual noise levels in the reconstructed signal, in the case of NA-MEMD, remained relatively stable with an increase in the input noise power. The power of added noise relative to the input signal power is an important factor in both EEMD and NA-MEMD since it affects the extent to which a quasi-dyadic filter bank structure is imposed on input data. In EEMD, this issue is more critical since noise is added directly to the signal.

Also observe from Fig. 5 that the power of reconstruction error function corresponding to NA-MEMD had significantly smaller values as compared to that of EEMD (note the difference in the ranges of $y$-axis in the two subfigures), because of negligible residual noise in the case of NA-MEMD. The error function in EEMD had larger values due to high levels of residual noise caused by directly adding noise to the input signal.

In the simulations, within EEMD, $N=1000$ realizations of WGN were used and the noise power was varied in the range of $0.01-1$ (1-100\% of the original data power), whereas, for NA-MEMD, $l=2$ WGN channels were added with the same noise power as was used in EEMD. The number of direction vectors used in MEMD was $V=64$.

\subsection{Effect of the number of noise channels in NA-MEMD on residual noise}

Another important aspect of NA-MEMD is how the number of noise channels $l$ affects the residual noise levels. In the simulations presented in this paper, we used 


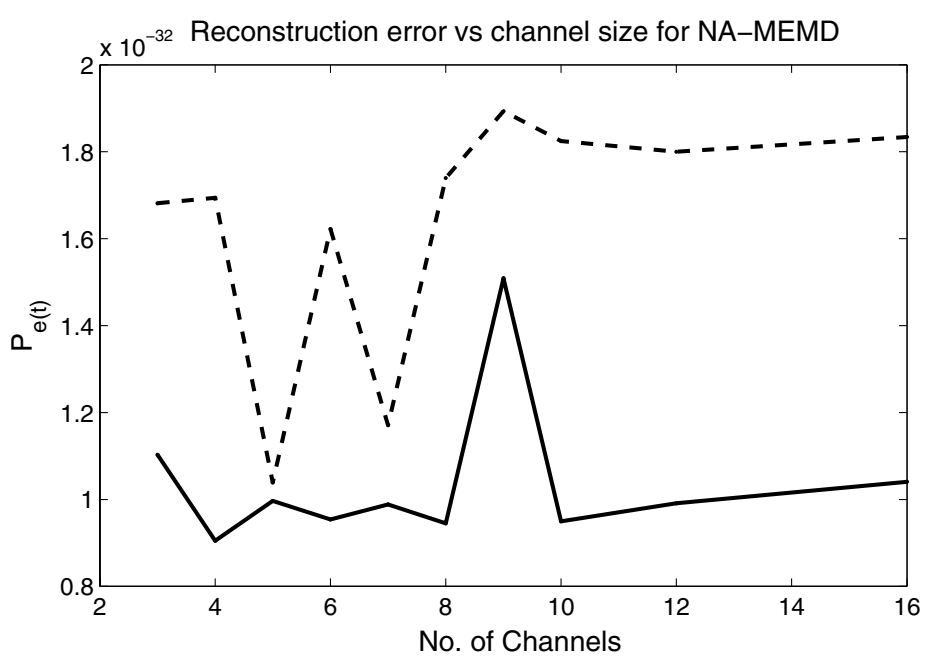

Fig. 6. Power of the reconstruction error signal, obtained using NA-MEMD, plotted as a function of number of WGN channels, $l$, for the input signals in Fig. 3 (dashed line) and Fig. 4 (thick line).

$l=2$, however there is no limit on the number of noise channels. To give an empirical estimate of the dependence of NA-MEMD on the number of noise channels, simulations were performed on the signals, shown in Figs. 3 and 4, by varying the number of noise channels $l$ and calculating the power of the reconstruction error. ${ }^{f}$ The results in Fig. 6 show that the reconstructed error is negligible, even for larger $l$, ensuring the completeness of NA-MEMD.

\section{Applications in EEG Signal Processing}

Many EEG applications estimate signals belonging to different frequency bands; for instance, voluntary limb movements cause changes in the $m u$ and beta rhythms observed in the central region of the brain, which need to be effectively isolated for advanced BCI applications [Pfurtscheller et al. (1997)]. The EMD-based algorithms, being data-driven, are highly suitable for nonstationary data and, thus, are a natural choice for their analysis. Mode-mixing, however, presents a major obstacle to the accurate data analysis, as it prevents the EEG frequency bands from being localized.

We next present simulation results obtained by applying NA-MEMD to real world multichannel EEG signals, together with a comparative analysis of standard univariate EMD and EEMD applied channel-wise to the EEG data. The two classes of EEG signals considered were: steady SSVEP responses and motor imagery EEG data. The EEG data sets are multivariate and for such data the NA-MEMD method, by design, was expected to perform better than the univariate EMD and

\footnotetext{
${ }^{\mathrm{f}}$ In the simulation, the noise power in the two channels was kept at $0.05, N=200$ noise ensembles were employed, and the number of direction vectors used in MEMD algorithm were $V=64$.
} 
EEMD algorithms applied channel-wise. In addition to direct multi-channel data processing, we also applied the NA-MEMD algorithm to single-channel EEG data and compared its performance against the standard EMD algorithms.

For comparative analysis between standard EMD, EEMD, and NA-MEMD, to reflect mode-mixing issues, we plotted the power spectra of corresponding IMFs; clear separability and less overlapping of IMFs (corresponding to different EEG bands) in the spectrum would mean less mode-mixing and, hence, improved frequency localization and better overall performance. In the simulations, we chose two-dimensional noise subspace, $l=2$, with each channel having a noise power of 0.05; all EEG data channels were first normalized to have a unit variance. The number of directions used in the MEMD algorithm was $V=64$ and the parameters of the stopping criterion used were $\left[\sigma_{1}=0.075, \sigma_{2}=0.75, \alpha=0.075\right]$.

\subsection{SS VEP data processing}

Electroencephalogram (EEG), the most convenient means to measure neurophysiological activity due to its noninvasive nature and affordable recording equipment, is commonly used to record SSVEP. Since the SSVEP data exhibits electrical activity at the same (and multiples of) frequency of the visual stimulus, it is natural to apply EMD-based methods to accurately extract the modes corresponding to the applied stimulus.

In the simulations, we considered 7 EEG channels from the subject who was presented with a visual blinking signal at $15 \mathrm{~Hz}$. We first applied NA-MEMD (directly) and EMD (channel-wise) on such 7-channel input data, and the results are shown respectively in Figs. 7(a) and 7(b). It can be noticed that applying EMD separately on each EEG channel resulted in the mode-mixing and mode-misalignment in the corresponding IMFs, as evidenced by the overlapping of spectra of different IMFs from multiple input channels. Specifically, both IMF4 and IMF5 contributed to the mode corresponding to visual stimulus at $15 \mathrm{~Hz}$. Moreover, spectra of IMF5 and IMF6 from different channels overlap, resulting in further mode-mixing. On the other hand, application of NA-MEMD to the same data set resulted in IMFs which were aligned in frequency, each containing only a single temporal mode, as shown in Fig. 7(b), where the IMF6 contains the $15 \mathrm{~Hz}$ SSVEP response.

Next, both EMD and NA-MEMD were applied to single-channel SSVEP data to illustrate the advantages in computing univariate EMD via NA-MEMD. A trend similar to the above was observed as the IMF spectra obtained from NA-MEMD revealed very little mode-mixing, as shown in Fig. $7(\mathrm{~d}$ ) (a single IMF for $15 \mathrm{~Hz}$ SSVEP response), while the EMD-based spectrum shown in Fig. 7(c) shows modemixing in IMF5 and IMF6 for the same component. As a result, the IMF5 which corresponds to the $15 \mathrm{~Hz}$ visual stimulus had comparatively less power than the corresponding IMF from NA-MEMD. The time plots for the decomposition of a single channel SSVEP data by EMD and NA-MEMD are shown in Figs. 8(a) and 8(b) respectively; in the EMD-based decomposition, the mode-mixing is evident in IMF4 


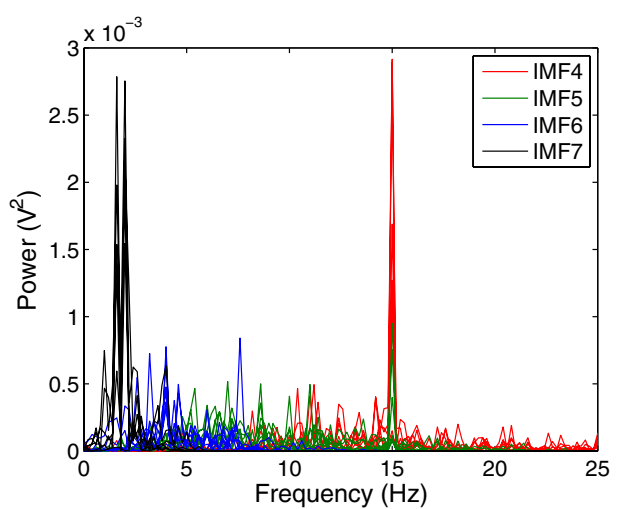

(a) EMD spectra of SSVEP data

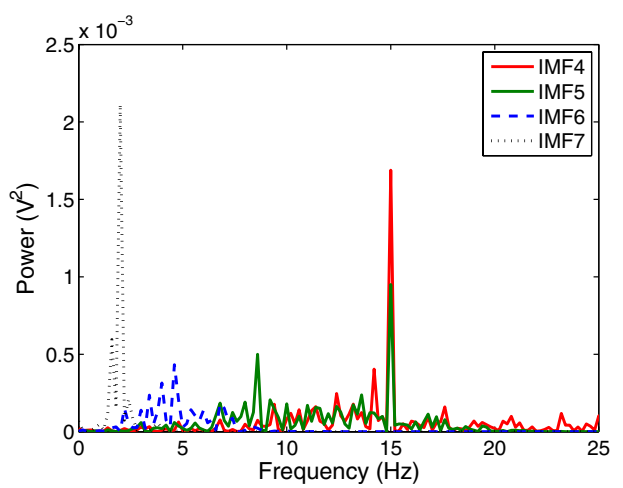

(c) EMD of a single-channel SSVEP

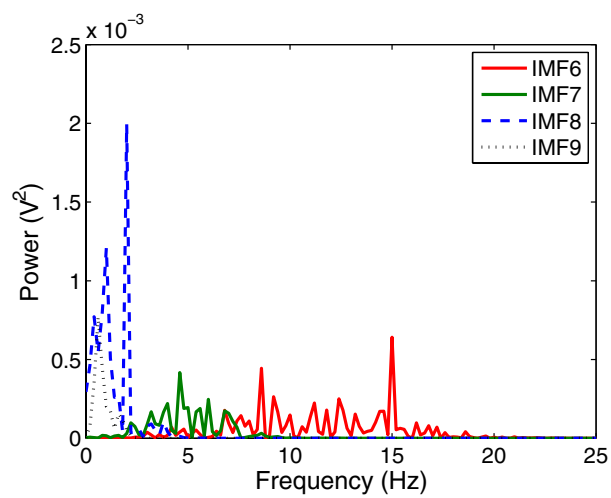

(e) NA-MEMD for small noise amplitude

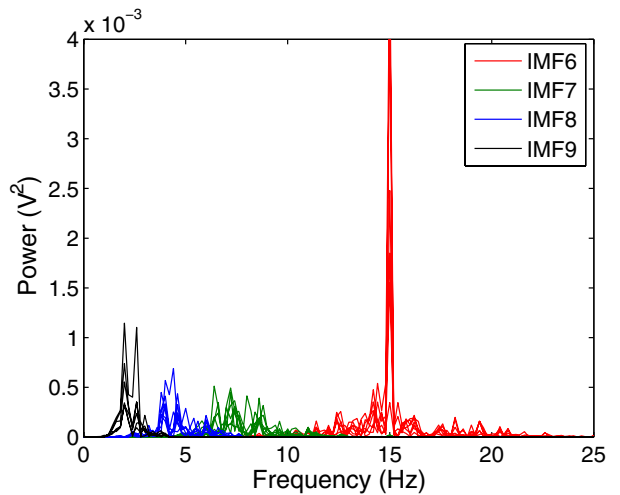

(b) NA-MEMD spectra of SSVEP data

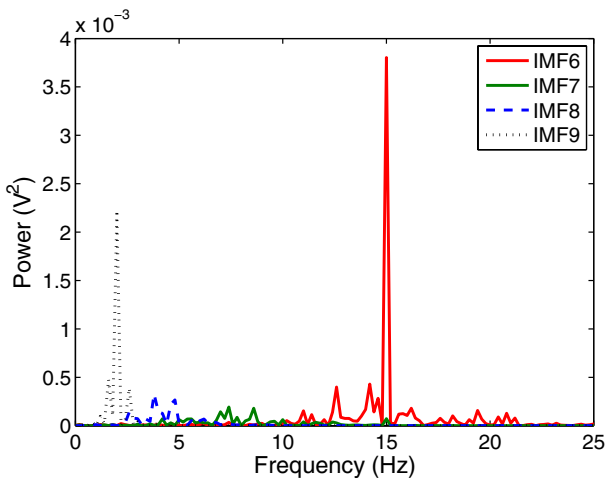

(d) NA-MEMD of a single-channel SSVEP

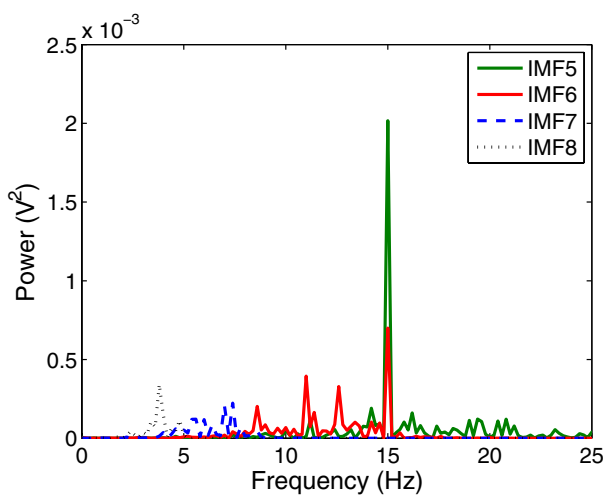

(f) NA-MEMD for large noise amplitude

Fig. 7. (Color online) Power Spectra of IMFs obtained by applying EMD and NA-MEMD on a multivariate (7-channel) and a single channel SSVEP EEG data. The effects of noise amplitudes on NA-MEMD output spectrum for relatively (e) lower and (f) larger noise powers. 

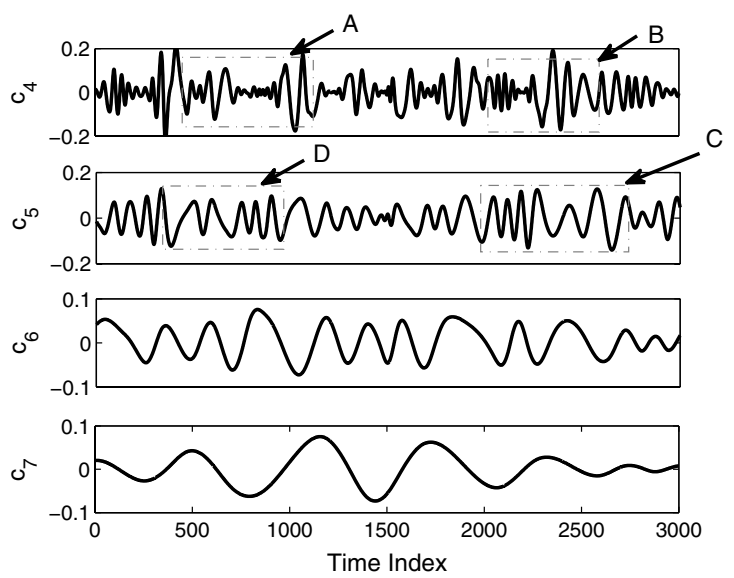

(a) Time decomposition of SSVEP data by EMD

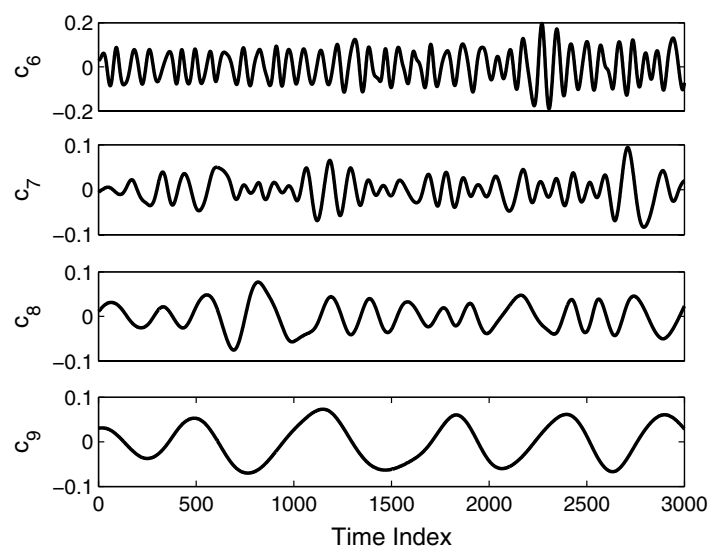

(b) Time decomposition of SSVEP data by NA-EMD

Fig. 8. Waveforms for single-channel decomposition of SSVEP EEG data, using (a) EMD and (b) NA-MEMD. Observe the mode-mixing in IMF4 and IMF5 in EMD-based time plots, as identified by boxes labelled as $A, B, C$, and $D$. IMFs obtained from NA-MEMD, on the other hand, contain a single mode only and, thus, are free from mode-mixing.

and IMF5, particularly at locations highlighted in dashed rectangular boxes, while all the components (IMFs) of NA-MEMD contained only a single mode, free from mode-mixing.

As mentioned earlier, choosing suitable amplitudes for the $l$ noise channels is a prerequisite for the successful operation of NA-MEMD. In Figs. 7(e) and 7(f), we show two cases in which comparatively lower $(0.5 \%$ of the input noise power) and higher ( $25 \%$ of the input noise power) noise levels were used in NA-MEMD for a single-channel SSVEP data. Employing lower noise levels tends to reduce the power of the mode corresponding to the visual stimulus (IMF5), a trend similar to 
that observed in the case of standard EMD (see Fig. 7(c)); this was expected as standard EMD can be considered as a special case of NA-MEMD for infinitesimally small input noise levels. For higher noise amplitudes, mode-mixing is clearly visible in the mode corresponding to the visual stimulus, as shown in Fig. 7(f). This can be attributed to the loss of data-driven nature of NA-MEMD due to excessive input noise power.

\subsection{Motor imagery data processing}

Motor imagery tasks refer to the imagination of a motor action without any actual movement of limbs. They have clear practical significance in EEG-based BCI applications; for instance, EEG data from imaginary mental tasks can be used to control a mechanical device through brain-machine interface. The neurophysiological basis for motor imagery BCI are the so-called mu $(8-12 \mathrm{~Hz})$ and beta rhythms (18$25 \mathrm{~Hz}$ ) [McFarland et al. (2000)], which have been observed in the central region of the brain, using EEG, when subjects plan and execute hand or finger movements [Pfurtscheller and da Silva (1999)]. The so observed changes to the $m u$ and beta rhythms have been extracted using the EMD method for the classification of bistable perception [Wang et al. (2008)]. In this section, we illustrate the ability of NA-MEMD to obtain enhanced localization of the frequency information in motor imagery EEG data and compare the results with those obtained from standard EMD and EEMD methods.

The BCI Competition IV Dataset I was used for the following analysis ${ }^{\mathrm{g}}$; it was recorded from four healthy subjects using 59 electrodes sampled at $1,000 \mathrm{~Hz}$ [Blankertz et al. (2007)]. Each subject selected two motor imagery tasks among three: left hand, right hand, and foot (both feet). Subjects performed a total of 200 trials. In each trial, the subject imagined one of the two possible tasks (one task per trial) for a duration of $4 \mathrm{~s}$. Out of the available 59 EEG channels, 11 were selected for analysis: "FC3", "FC4", "Cz", "C3", "C4", "C5", "C6", "T7", "T8", "CCP3" and "CCP4", since the motor imagery response is primarily associated with the central area of the brain [Pfurtscheller and da Silva (1999)].

The data was processed using the EMD, EEMD, and NA-MEMD algorithms and the average power spectra of relevant IMFs $c_{1}(t)-c_{4}(t)$ are plotted in Fig. 9 for the two subjects; the averaging was performed over all 200 trials on each subject. The power spectrum plots were used to gauge the performance of NA-MEMD, relative to standard EMD and EEMD methods, with regards to the localization of different frequency rhythms ( $m u$ and beta). Clear separability (accurate localization) of the modes corresponding to $m u$ and beta rhythms would facilitate the accurate classification of motor imagery tasks through feature extraction algorithms. ${ }^{\mathrm{h}}$

${ }^{\mathrm{g}}$ The data set and its complete detail are available for downloading at: http://www.bbci.de/ competition/iv/.

${ }^{\mathrm{h}}$ It is not our purpose here to report the improvement in the final classification of EEG-based motor imagery tasks achieved via NA-MEMD; that work is currently under process. 

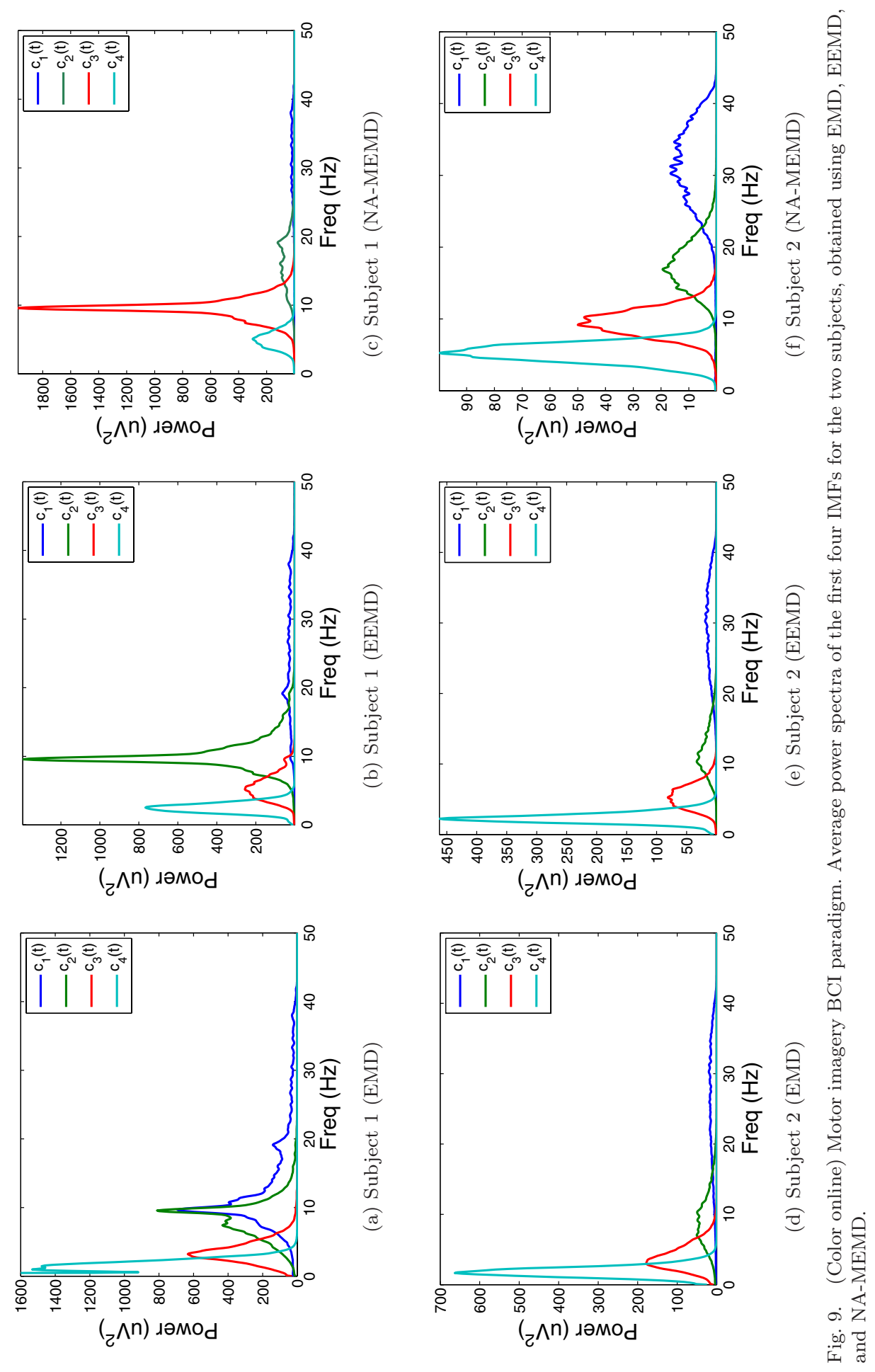
Figure 9 compares the average frequency distributions at the IMF level using standard EMD, EEMD, and NA-MEMD. Notice that IMFs obtained from EEMD and NA-MEMD were more localized in frequency, and that greater separation exists between their IMF frequency distributions. This indicates a greater consistency in the pattern of extracted EEG components between trials, and that these components have been better separated. In particular, the $m u$ and beta rhythms exhibit clear separation using NA-MEMD, contained in the IMFs $c_{2}(t)$ and $c_{3}(t)$. Using the univariate EEMD, these two different rhythms were extracted erroneously within a single IMF component, $c_{2}(t)$, illustrating poorer separability compared to the multichannel NA-MEMD algorithm. Observe that the different frequency ranges corresponding to the IMFs of NA-MEMD were also more consistently distributed across the subjects than those obtained from EMD and EEMD.

\section{Discussion}

We have shown that the improvements offered by NA-MEMD, in terms of reduced residual noise in the output, lower sensitivity to added noise power and enhanced frequency localization, make it a viable alternative to both EMD and EEMD when processing real world EEG data. It should be mentioned that the noise-assisted methods, including both EEMD and NA-MEMD, are expected to be useful for signals in which the dyadic filter bank decomposition may be relevant. That is, if the desired signal resides in multiple dyadic sub-bands, then choosing the noise-assisted methods for decomposition may even "spread" the desired signal across multiple IMFs, resulting in unwanted mode-mixing. In those cases, enforcing a dyadic filter bank structure on the input signal may reduce the inherent data-driven ability of EMD-based algorithms. For instance, the sharpness of the NA-MEMD filter due to its quasi-dyadic filter bank nature has been found to strip the harmonics off from the intrinsic nonlinear waves, such as the Duffing waves. However, in most cases, this problem can be remedied by an additional rectification step of combining two components into a single component. For the same reason, it has been observed that the performance of NA-MEMD deteriorates with an increase in the noise power, above $10 \%$ relative to the original signal (See Fig. $7(\mathrm{f})$ ). Our analysis suggests that the optimal noise levels for NA-MEMD are in the range of $2-10 \%$ of the input data power (variance).

We also encountered instances where NA-MEMD did not offer improvement or even performed worse than the standard EMD approach; for instance, in the classical case of two-tone signals, that is, signals containing sum of two sinusoids. Such signals consist of pure oscillatory components which are already IMFs within standard EMD, by its very design. Adding noise channels to such data, as in the NA-MEMD method, makes it hard for the algorithm to accurately extract the pure oscillatory components. A similar trend is expected in the case of multivariate signals consisting of pure rotational components. However, most real world signals, 
such as EEG data rarely exhibit pure oscillations or rotations and are, thus, a perfect match for NA-MEMD, as shown in Sec. 5 .

\section{Conclusions}

We have advocated the usefulness of calculating EMD using NA-MEMD. The method has been shown to be flexible and to yield physically meaningful components of the decomposition, whereby the power of added noise controls the operation of the algorithm. The mode-alignment and the filter bank property of NA-MEMD have been illustrated through examples, and the equivalent impulse response of the algorithm has also been given. Illustrative examples and performance evaluations have been provided to demonstrate the usefulness of the NA-MEMD method in comparison with the standard EEMD method, in terms of frequency localization, noise interference, and mode-mixing. Finally, the advantage of NA-MEMD has been demonstrated in the extraction and localization of frequency signatures in applications related to BCI based on EEG recordings.

It should be pointed out that in many applications the multivariate signal processing approach is highly desirable and of crucial importance. For instance, EEG data is inherently multivariate and exhibits significant coupling between its different channels. To obtain physically meaningful interpretation from such data sets, it is therefore desirable to decompose all the variables observed into the same number of IMF components of comparable scales. In such cases, the application of NA-MEMD yields great advantages over the univariate EMD and EEMD methods applied channel-wise.

\section{Appendix A. Low-Discrepancy Hammersley Sequences and MEMD Algorithm}

The discrepancy can be seen as a measure of irregularity of the distribution, or in other words, a quantitative measure for the deviation from the uniform distribution. This is important in quasi-Monte Carlo methods which are used to generate well-conditioned deterministic point sets for solving numerical integration problems.

The discrepancy $D_{K}(\mathcal{B} ; P)$ of a point set $P$ containing $K$ points, consisting of $x_{1}, x_{2}, \ldots, x_{K} \in I_{s}$ where $I_{s}$ is the closed $s$-dimensional unit cube, is given by

$$
D_{K}(\mathcal{B} ; P)=\sup _{B \in \mathcal{B}}\left|\frac{A(\mathcal{B} ; P)}{K}-\lambda_{s}(B)\right|,
$$

where $\mathcal{B}$ is a family of Lebesgue-measurable subsets of $I_{s}$ and $A(\mathcal{B} ; P)$ is the number of elements of the set $x_{1}, x_{2}, \ldots, x_{K} \in I_{s}$ in $\mathcal{B}$. The resulting value of $D_{K}$ is always between $0 \leq D_{K}(\mathcal{B} ; P) \leq 1$. 
The error analysis of the quasi-Monte Carlo integration shows that the error bounds are small for point sets with low discrepancy measure. This leads to an informal definition of the low-discrepancy sequences, as those having lower value of $D_{K}(\mathcal{B} ; P)$.

A positive integer $n \geq 0$, can be represented in base $b$ using

$$
n=\sum_{j=0}^{L-1} a_{j}(n) b^{j}
$$

For an integer $b \geq 2$, a radical inverse function $\phi_{b}$ in base $b$ can then be defined as

$$
\phi_{b}(n)=\sum_{j=0}^{L-1} a_{j}(n) b^{-j-1} \quad \text { for all } n \geq 0 .
$$

The van der Corput sequence in base $b$ is the sequence $x_{1}, x_{2}, \ldots, x_{L-1}$ with $x_{n}=$ $\phi_{b}(n)$ for all $n \geq 0$.

Let $s \geq 1$ be the given dimension and $b_{1}, \ldots, b_{s}$ be arbitrary coprime integers $\geq 2$. Then the Halton sequence in the bases $b_{1}, \ldots, b_{s}$ can be defined as the sequence $\mathbf{x}_{1}, \mathbf{x}_{2}, \ldots$ with

$$
\mathbf{x}_{n}=\left(\phi_{b_{1}}(n), \ldots, \phi_{b_{s}}(n)\right) \text { for all } n \geq 0 \text {. }
$$

Let $b_{1}, \ldots, b_{s-1}$ be the coprime positive integers $\geq 2$. For a given value of $s$ and $K$, the multi-dimensional Hammersley set of size $K$ is defined as

$$
\mathbf{x}_{n}=\left(\frac{n}{K}, \phi_{b_{1}}(n), \ldots, \phi_{b_{s-1}}(n)\right) \quad \text { for } n=0, \ldots, K-1 .
$$

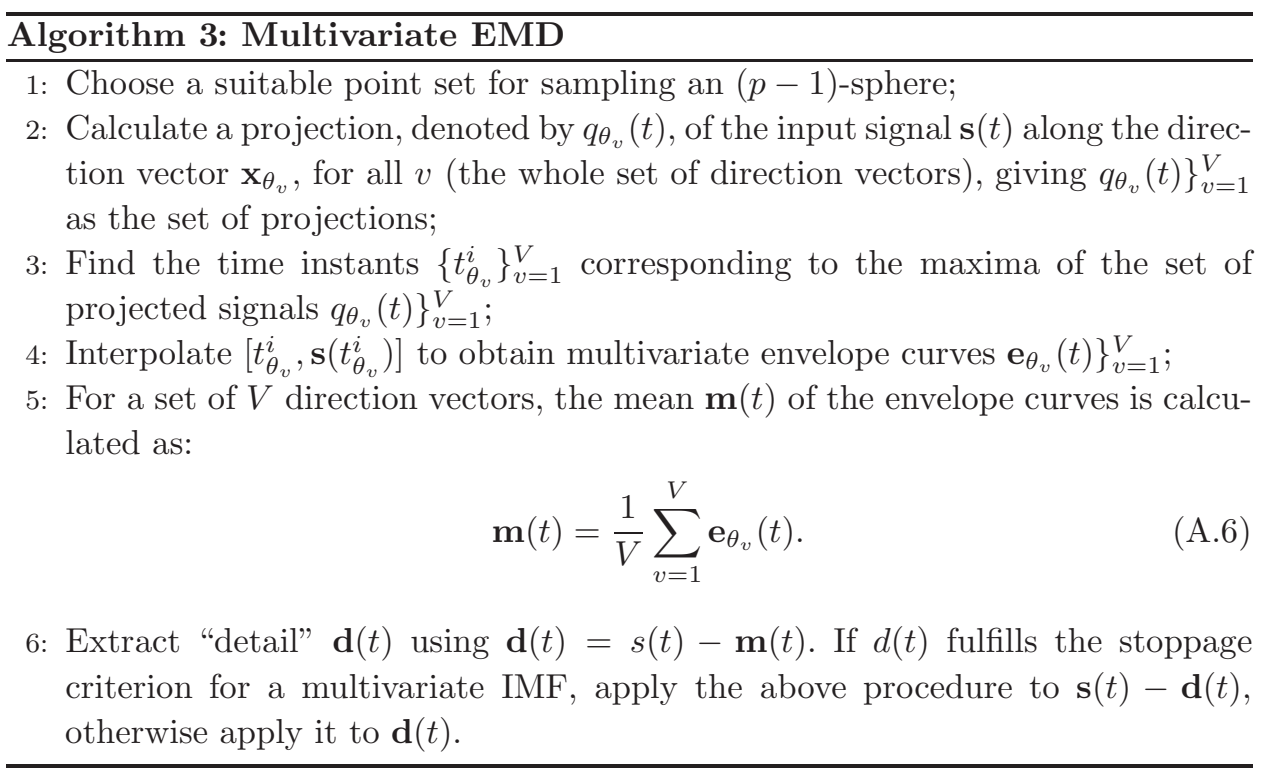


To generate the uniform samples on a general $n$-sphere, a linear mapping to $(n-1)$ angular coordinates is first performed, and then the direction vectors based on these coordinates are generated.

Consider a sequence of $p$-dimensional vectors $\mathbf{s}(t)=\left\{s_{1}(t), s_{2}(t), \ldots, s_{p}(t)\right\}$, representing a multivariate signal with $p$ channels, and the symbol $\mathbf{x}_{\theta_{v}}=$ $\left\{x_{1}^{v}, x_{2}^{v}, \ldots, x_{p}^{v}\right\}$ denoting a set of $v=1,2, \ldots, V$ direction vectors along the directions given by angles $\theta_{\mathbf{v}}=\left\{\theta_{v_{1}}, \theta_{v_{2}}, \ldots, \theta_{v_{p-1}}\right\}$ in $\mathbb{R}^{p}$. Then, the steps for obtaining signal decomposition via MEMD are summarized in Algorithm 3.

\section{References}

Altaf, M. U., Gautama, T., Tanaka, T. and Mandic, D. P. (2007). Rotation invariant complex empirical mode decomposition. Proceedings of the IEEE International Conference on Acoustics, Speech, Signal Processing, Vol. 3, Honolulu, pp. 1009-1012.

-Blankertz, B., Dornhege, G., Krauledat, M., Muller, K. and Curio, G. (2007). The noninvasive Berlin brain-computer interface: Fast acquisition of effective performance in untrained subjects. NeuroImage, 37: 539-550.

Cui, J. and Freeden, W. (1997). Equidistribution on the sphere. SIAM J. Scient. Comput., 18: 595-609.

-Flandrin, P. and Goncalves, P. (2004). Empirical mode decomposition as data-driven Wavelet like expansions. Int. J. Wave. Multires. Inform. Process., 2: 1-20.

Flandrin, P., Rilling, G. and Goncalves, P. (2005). EMD equivalent filter banks, from interpetation to applications, Chap. 3, 1st edn. Hilbert-Huang Transform and Its Applications. World Scientific, Singapore, pp. 67-87.

-Huang, N. E., Shen, Z., Long, S., Wu, M., Shih, H., Zheng, Q., Yen, N., Tung, C. and Liu, H. (1998). The empirical mode decomposition and Hilbert spectrum for non-linear and non-stationary time series analysis. Proc. Royal Soc. A, 454: 903-995.

-Huang, N. E., Wu, Z., Long, S. R., Arnold, K. C., Chen, X. and Blank, K. (2009). On instantaneous frequency. Adv. Adapt. Data Analysis, 1: 177-229.

Looney, D. and Mandic, D. P. (2009). Multi-scale image fusion using complex extensions of EMD. IEEE Trans. Signal Process., 57: 1626-1630.

Mandic, D. P. and Goh, V. S. L. (2009). Complex Valued Non-Linear Adaptive Filters: Noncircularity, Widely Linear Neural Models. Wiley, United Kingdom.

- McFarland, D. J., Miner, L. A., Vaughan, T. M. and Wolpaw, J. R. (2000). Mu and beta rhythm topographies during motor imagery and actual movements. Brain Topograp., 12: $177-186$.

Niederreiter, H. (1992). Random Number Generation and Quasi-Monte Carlo Methods. Society for Industrial and Applied Mathematics (SIAM), Philadelphia, Pennsylvania.

Pfurtscheller, G. and da Silva, F. H. L. (1999). Event-related EEG/MEG synchronization and desynchronization: Basic principles. Clinical Neurophysiol., 110: 1842-1857.

-Pfurtscheller, G., Neuper, C., Flotzinger, D. and Pregenzer, M. (1997). EEG-based discrimination between imagination of right and left hand movement. Electroencephalograp. Clinical Neurophysiol., 103: 642-651.

-Rehman, N. and Mandic, D. P. (2010a). Empirical mode decomposition for trivariate signals. IEEE Trans. Signal Process., 58: 1059-1068.

-Rehman, N. and Mandic, D. P. (2010b). Multivariate empirical mode decomposition. Proc. Royal Soc. A, 466: 1291-1302.

-Rehman, N. and Mandic, D. P. (2011). Filterbank property of multivariate empirical mode decomposition. IEEE Trans. Signal Process., 59: 2421-2426. 
Rilling, G., Flandrin, P. and Goncalves, P. (2003). On empirical mode decomposition and its algorithms. IEEE-EURASIP Workshop Nonlinear Signal Image Processing (NSIP), Trieste, Italy.

-Rilling, G., Flandrin, P., Goncalves, P. and Lilly, J. M. (2007). Bivariate empirical mode decomposition. IEEE Signal Process. Lett., 14: 936-939.

Torres, M. E., Colominas, M. A., Schlotthauer, G. and Flandrin, P. (2011). A complete ensemble empirical mode decomposition with adaptive noise. Proceedings of the IEEE International Conference on Acoustics, Speech and Signal Processing, Prague, pp. 4144-4147.

Wang, Z., Maier, A., Logothetis, N. K. and Liang, H. (2008). Single-trial classification of bistable perception by integrating empirical mode decomposition, clustering, and support vector machine. EURASIP J. Advan. Signal Process., Vol. 2008, 1-8.

-Wu, Z. and Huang, N. E. (2009). Ensemble empirical mode decomposition: A noise-assisted data analysis method. Advan. Adapt. Data Anal., 1: 1-41.

-Yeh, J.-R., Shieh, J.-S. and Huang, N. (2010). Complementary ensemble empirical mode decomposition: A novel noise enhanced data analysis method. Advan. Adapt. Data Anal., 2: 135-156. 